\title{
Menaces D'origine Anthropique Et Habitat De Pan Paniscus Dans La Reserve Naturelle De Sankuru, En Republique Democratique Du Congo
}

\author{
Nyembo Faustin, MA \\ Doctorant en Changement Climatique Biodiversité et Agriculture Durable \\ CCBAD à l'Université Félix Houphouët-Boigny, Abidjan, Côte d'Ivoire. \\ Chercheur au Département de Zootechnie, Université Officielle de \\ Mbujimayi, République Démocratique du Congo

\section{Mertens Benoit, PhD} \\ UMR Espace Dev, Institut de Recherche pour le Développement IRD, \\ Maison de la télédétection, Montpellier Cedex France \\ Cherif Mamadou, PhD \\ Inza Koné, PhD \\ UFR Biosciences, \\ Université Félix Houphouët-Boigny, Abidjan, Côte d'Ivoire
}

Doi:10.19044/esj.2020.v16n21p290 URL:http://dx.doi.org/10.19044/esj.2020.v16n21p290

\section{Résumé}

Le bonobo (Pan paniscus), est une espèce endémique à la République Démocratique du Congo (RDC). La présente étude identifie les menaces qui pèsent sur ce primate au niveau de la Réserve Naturelle de Sankuru (RNSA), les priorise à partir des analyses spatiales en évaluant, de manière comparative, l'évolution de l'occupation du sol à l'intérieur et extérieur de la zone protégée. Par rapport au reste de l'aire de répartition historique du bonobo en RDC où la chasse est une préoccupation majeure, les enquêtes dans la zone d'étude révèlent que l'agriculture sur brûlis est la première menace d'origine anthropique qui influe plus sur la survie de ce primate. L'étude révèle des signes d'existence d'une ignorance persistante aux lois du pays en matière de gestion et de conservation de la nature de la part de la population locale. La croissance démographique, le manque d'alternatives de subsistance ainsi que l'expansion de l'occupation du sol à l'intérieur et aux alentours de la RNSA présentent des grandes envergures spatiales et un impact très remarquable sur le couvert végétal. La vitesse de l'expansion du complexe rural est de 1,5\% dans la RNSA soit 3600 ha/an. Cette extension se fait de façons identiques à l'intérieur et à l'extérieur de la limite de la RNSA. 
Mots-clés: Biodiversité, aire protégée, menace, Bonobo

\title{
Anthropogenic Threats and Pan Paniscus Habitat in the Sankuru Nature Reserve, Democratic Republic of Congo
}

\author{
Nyembo Faustin, MA \\ Doctorant en Changement Climatique Biodiversité et Agriculture Durable \\ CCBAD à l'Université Félix Houphouët-Boigny, Abidjan, Côte d'Ivoire. \\ Chercheur au Département de Zootechnie, Université Officielle de \\ Mbujimayi, République Démocratique du Congo \\ Mertens Benoit, PhD \\ UMR Espace Dev, Institut de Recherche pour le Développement IRD, \\ Maison de la télédétection, Montpellier Cedex France \\ Cherif Mamadou, PhD \\ Inza Koné, PhD \\ UFR Biosciences, \\ Université Félix Houphouët-Boigny, Abidjan, Côte d'Ivoire
}

\begin{abstract}
The bonobo (Pan paniscus), is an endemic species to the Democratic Republic of Congo (DRC). This study identifies the threats to this primate in the Sankuru Natural Reserve (RNSA), prioritizes them based on spatial analyzes by evaluating, in a comparative manner, the land use evolution inside and outside the protected area. Compared to the rest of the bonobo distribution area where hunting is a major concern, surveys reveal that slash-and-burn agriculture is the first anthropogenic threat that has the greatest impact on bonobo's survival in the RNSA. The reveals signs of persistent ignorance to the country's laws on the management and conservation of nature by the local population. Population growth, the lack of subsistence alternatives as well as the expansion of the rural complex within and around the RNSA present large spatial dimensions and a very remarkable impact on the vegetation cover. The rate of expansion of the rural complex is $1.5 \%$ in the RNSA or 3600 ha / year. This extension of the rural complex is done in identical ways inside and outside the RNSA boundary.
\end{abstract}

Keywords: Biodiversity, Protected Area, Threat, Bonobo 


\section{Introduction}

La biodiversité du bassin du Congo englobe des paysages diversifiés allant des forêts d'altitude aux forêts denses humides et aux zones de savanes (Akaibe, 2014). La République démocratique du Congo (RDC) est l'un des six pays qui constituent le bassin du Congo ; elle possède une diversité biologique exceptionnelle qui fait d'elle, l'un des 18 pays de mégadiversité à l'échelle mondiale (Mittermeier et al., 2008 ; Doumenge et al., 2015). Parmi cette exceptionnelle diversité, il y a la présence de plus de genres de primates que tous les pays du monde, dont trois genres de 4 grands singes notamment, le Gorilla beringei beringei, le Pan troglodytes schweinfurthii et le Pan paniscus (MEDD, 2016). Malgré la forte pression qui s'y exerce, le pays renferme encore des espèces emblématiques. Cependant, il n'est un secret pour personne que depuis la révolution industrielle, la biodiversité n'a cessé de décroitre. A ce jour, le rythme d'extinction des espèces est environ mille fois supérieur à ce qu'il était par le passé et il est probable que ce rythme actuel soit multiplié par dix dans le futur (Vos et al., 2014; Coutu, 2016).

De multiples facteurs permettent d'expliquer cette perte de biodiversité notamment la dégradation des habitats, le braconnage et la surexploitation des écosystèmes forestiers pour approvisionner des principales villes et des bourgs diamantifères en charbon de bois et en produits forestiers non ligneux (feuilles, fruits sauvages, tubercules et autres éléments tels que les chenilles, champignons, etc.) (Kabata, 2018). Ces facteurs sont amplifiés dans la plupart des cas enregistrés en Afrique, par des instabilités politiques entrainant des augmentations spectaculaires de l'empiétement dans des forêts supposées protégées (Gonedelé et al., 2006).

La présente étude s'intéresse aux interactions entre menaces d'origine anthropique et habitats des primates, particulièrement du bonobo (Pan paniscus), une espèce de primate endémique à la RDC qui vit au plus profond de la forêt du bassin du Congo, dans une zone relativement peu perturbée (Eriksson et al., 2004). Ce grand singe est classé En Danger à cause du braconnage et du commerce de viande de brousse (Fruth et al., 2016). L'objectif de l'étude est d'analyser, à l'échelle de la RNSA, les causes potentielles d'origine anthropiques susceptibles de réduire l'aire de répartition du bonobo. La RNSA est la réserve la mieux adaptée pour cette étude. En effet, le bonobo fait partie de la valeur de cette réserve, c'est-à-dire de l'ensemble des éléments que cherche à conserver cette aire protégée (Mauvais et al., 2017). En outre, la RNSA est la plus exposée à la montée progressive des menaces d'origine anthropique, partant du sud du pays vers le nord. Plusieurs ateliers rapportés par Thompson et al. (1995) et Coxe et al. (2000) ainsi que des publications scientifiques antérieures (Thompson et al. 2003; GRASP 2005) ont évoqué les questions liées aux menaces qui planent sur les bonobos. Cependant, la plupart de ces travaux étaient jugés trop globaux (UICN et 
ICCN, 2012) ; les questions étaient beaucoup plus analysées à l'échelle de l'aire de répartition historique du bonobo d'une superficie d'environ 564.542 $\mathrm{km}^{2}$ (Figure 9). Selon Narat et al. (2012) la conservation d'espèces charismatiques telles que le chimpanzé (Pan troglodytes) et le bonobo (Pan paniscus) et de leurs habitats, implique de mieux comprendre leur adaptabilité à l'anthropisation du milieu, afin d'adopter des stratégies de conservation efficaces et durables qui prennent en compte les besoins et les traditions des populations locales. En outre, l'évaluation de l'intégrité du couvert forestier des aires protégées est capitale pour leur bonne gestion (Muyaya et al., 2016).

La présente étude est la première du genre à porter sur l'identification et la hiérarchisation des menaces qui pèsent sur le bonobo dans la RNSA d'une part et au suivi du couvert forestier de ladite réserve au cours des 30 dernières années.

\section{Matériel et méthodes}

\section{Présentation du site d'étude}

La zone d'étude composée de la Réserve Naturelle de Sankuru (RNSA) et d'une zone tampon (ZT). Cette zone tampon périphérique de la réserve n'a pas de statut légal de protection. D'une largeur de $50 \mathrm{~km}$, elle correspond à une zone de transition et intègre les alentours de la réserve. Créée en 2007, la RNSA fait partie des jeunes aires protégées de la RDC (Nyembo et al., 2019; FAO, 2020) pour la protection du bassin hydrographique du Sankuru et de la Lokenye en vue de garantir les débits des cours d'eaux du bassin du Congo et aussi pour la conservation d'une importante biodiversité animale comprenant spécifiquement le bonobo (Pan paniscus), l'okapi (Okapia johnstoni) et l'éléphant de forêt (Loxodonta-cyclotis). La RNSA couvre une superficie de $23161 \mathrm{~km}^{2}$ (UICN et ICCN, 2012; Doumenge et al., 2015). Elle est située au nord de la province de Sankuru à $-2,56^{\circ}$ de latitude et $24,15^{\circ}$ de longitude et appartient au paysage Salonga-Lukenie-Sankuru. La procédure de sa création est sujet de controverses (Liengola et al., 2009) et présentement, elle est la plus empiétée des aires protégées du Congo malgré la biodiversité qu'elle renferme (Mertens et Orekan, 2019).

\section{Méthode et outils}

La cartographie de l'occupation du sol et de son évolution, considérée comme un processus clé pour comprendre l'environnement (Chapman, 2005), a été utilisée dans ce travail pour analyser dans le temps et évaluer spatialement l'évolution de la distribution spatiale des impacts des phénomènes ou des menaces liées aux activités humaines sur le couvert forestier (Oliveau, 2011). L'envergure spatiale représente la proportion de l'habitat qui peut être négativement impactée par les menaces. Elle fait partie 
des critères utilisés par 1'ICCN pour la priorisation de menaces à l'échelle globale de l'aire de répartition historique de bonobo (UICN et ICCN, 2012).

Les données sont basées sur les alertes Glad de l'UMD et le Global Forest Watch du WRI qui fournissent des données récentes et fiables pour la surveillance et les pertes de forêts (Ickowitz et al., 2015; Molinario et al., 2015; Molinario et al., 2017). Ces données paraissent ces dernières années comme des méthodes intéressantes dans les forêts relativement humides d'Afrique centrale (Molinario et al., 2020). Elles sont complétées par les résultats des enquêtes menées dans le cadre du projet AGRNSA mis en œuvre avec l'appui de OSFACO (Observation Spatiale des Forêts d'Afrique Centrale et de l'Ouest) (Nyembo et al., 2019). L'avantage de données ciblées est qu'elles fournissent des cartes permettant de distinguer la perte de couverture forestière en se basant sur une mosaïque qui réunit toutes les différentes pertes dues à la présence des routes, des villages, des champs, des jachères dans les forêts qui supportent les activités humaines dans le pays. L'intérieur de cette mosaïque territoriale est appelé complexe rural. Il est décrit comme étant une mosaïque de couverture terrestre des routes, des rivières, des établissements humaines, des sols défrichés, champs cultivés et des forêts secondaires et primaires (Molinario et al., 2020). Pour cela, l'optimisation de données a nécessité l'exploitation de plusieurs bases de données cartographiques :

- Congo basin forest cover monitoring 1990-2000 (Hansen, 2008): https://glad.geog.umd.edu/dataset/congo-basin-forest-monitoringlandsat-1990-2000

- Global Forest Change 2000-2018 (Hansen, 2013, GFC: 2018): fournie les résultats de l'analyse chronologique des images Landsat caractérisant l'étendue et le changement de la forêt à l'échelle mondiale.

http://earthenginepartners.appspot.com/science-2013-global-forest

- Forest cover change, SNSF DRC, Forêt-Non forêt; 2000-2010 et 20102014

http://www.rdc-snsf.org

- Forest Cover Dynamics of Shifting Cultivation in the DRC: A remote sensing-based assessment for 2000-2010: fournie les données concernant la dynamique du couvert forestier de la culture itinérante en RDC, les fichiers utilisés sont en format: tif ; Projection: sinusoïdale ; Méridien central: 20 ; Système de référence: ArcInfo Sphere $(6370997 \mathrm{~m})$. Ces fichiers couvrent la RDC et sont utilisables dans tout système SIG (Molinario et al. 2015).

Les principales opérations telles que la superposition des données (couches); la création de vecteur (création d'une zone tampon et d'une grilles) ; l'analyse de raster (l'extraction de la zone d'étude « RNSA + ZT de 
$50 \mathrm{~km}^{2} \gg$, reclassification par table, histogramme zonale, statiques des zones) et la mise en page ont été réalisées avec le logiciel QGIS version 3.10, et exécutées conformément à la plus récente guide de l'utilisation (QGIS, 2020). Le logiciel Excel a été mis en contribution dans l'élaboration de différents graphiques de comparaison produits dans cette étude (Excel, 2016).

\section{Résultats}

Expansion du Complexe rural dans la RNSA et aux alentours (2000-20052010)

L'analyse spatiale de l'expansion du complexe rural autour de l'année de création de la RNSA en 2007 montre que la superficie du complexe rural au sein de la réserve augmente avant, pendant et après la création de cette aire protégée. En 2000 le complexe rural couvrait 13,9\% de la RNSA soit 3232,9 $\mathrm{km}^{2}$. Cinq ans après il atteint 14,6\% de la réserve et en 2010 sa superficie est estimée à $15,6 \%$. Entre 2000 et 2010 , l'équivalent de $360,5 \mathrm{~km}^{2}$ de forêt ont été convertis (Figures 1 et 2).

Par rapport à la zone tampon, le résultat obtenu montre qu'il existe de fortes pressions autour de la RNSA dans sa partie sud (figure 3). En 2010, le complexe rural occupait $33 \%$ de la zone tampon soit $15281 \mathrm{~km}^{2}$ (équivalant à $66 \%$ de la superficie totale de la RNSA) contre $14563 \mathrm{~km}^{2}$ en 2000 (Figure 4). L'analyse spatiale révèle que la vitesse de l'expansion du complexe est presque la même à l'intérieur de la zone protégée ainsi que dans la zone tampon. En 10 ans, le complexe rural s'est étendu de 1,559\% dans la RNSA soit 3600 ha/an et $1,553 \%$ dans la ZT.

\section{Dynamique spatiale du couvert végétal de 1990 à 2018 dans la RNSA}

Le suivi de la dynamique spatiale de la couverture forestière à l'intérieur de l'aire protégée de Sankuru ainsi qu'aux alentour entre 1990 et 2018, révèle que l'impact de l'agriculture sur l'habitat de bonobo de Sankuru est très remarquable ; il apparait sur la figure 5 que l'intensité de la pression sur le couvert forestier est trop élevée autour de la réserve. La perte du couvert forestier continue de progresser. Les statistiques présentées à la figure 6 montre une augmentation plus importante et rapide de la perte des forêts dans la zone tampon. En référence à l'année de la création de la réserve naturelle de Sankuru en 2007, la perte du couvert végétal dans la zone tampon est 7 fois plus importante en 2018. Au sein de la RNSA, la perte a quadruplet pendant la même période.

\section{Activités exercées par la population de Sankuru}

Le résultat sur les types activités exercées par la population de Sankuru montre que $50 \%$ des principales ethnies retrouvées dans la province de Sankuru pratiquent exclusivement l'agriculture ; $25 \%$ combinent l'agriculture 
et la chasse ; $12,5 \%$ pratiquent l'agriculture et la pêche ; une ethnie sur 16 ne pratique pas l'agriculture et $12,5 \%$ pratiquent tous les trois domaines d'activités à la fois (Figure 7). Les populations qui s'adonnent à l'agriculture, que cela soit de manière exclusive ou associée à d'autres activités, sont issues de 15 ethnies sur les 16 répertoriées (Figure 8). Aucune population ne pratique la chasse de manière exclusive.

Les enquêtes révèlent des effets positifs engendrés par des nombreuses contraintes concernant la chasse des espèces protégées. En rappel, de l'article 78 , alinéa $1^{\text {er }}$ de la loi ${ }^{\circ} 14 / 003$ du 11 février 2014 relative à la conservation de la nature en RDC stipule que la détention d'une espèce intégralement protégée est punie d'un an à dix ans d'emprisonnement et d'une amende de cinq millions à vingt millions de francs congolais. Cela rend difficile la vente des espèces protégées et décourage les braconniers et les acheteurs de Sankuru. Les récalcitrants font leurs commerces en cachète. La Photo 1 montre la carcasse d'un grand singe trouvée au prêt d'une vendeuse du marché central de Lomela à Sankuru. Contrairement aux autres viandes de chasse, la carcasse de ce grand singe n'était pas exposée sur les étalages du marché, elle était cachée dans un sac.
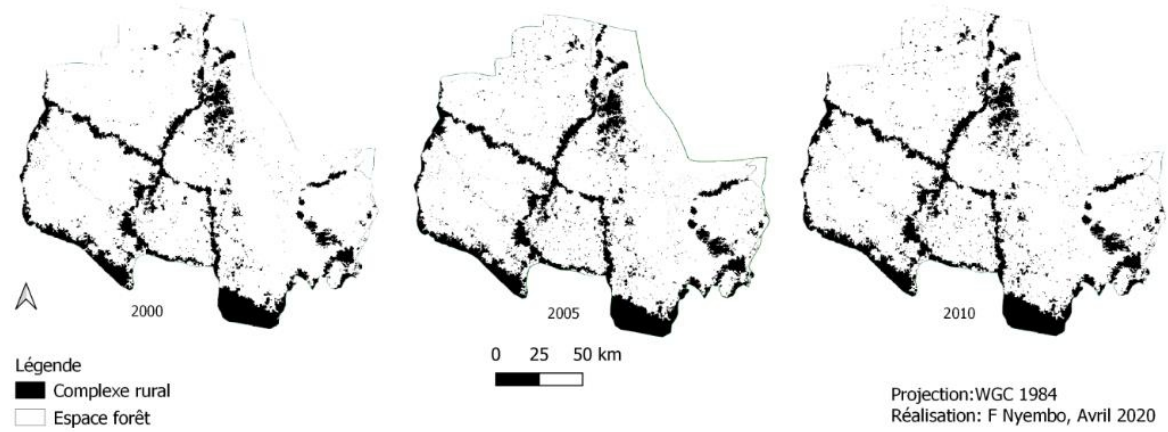

Figure 1 : Dynamique de la distribution du complexe rural dans la RNSA (Données : Forest Cover Dynamics of Shifting Cultivation in the DRC 2000-2010)

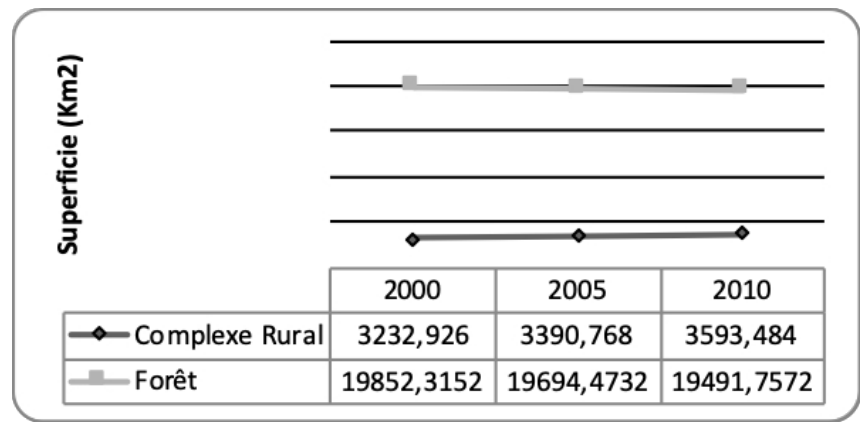

Figure 2 : Variation de la superficie du complexe rural dans la RNSA 

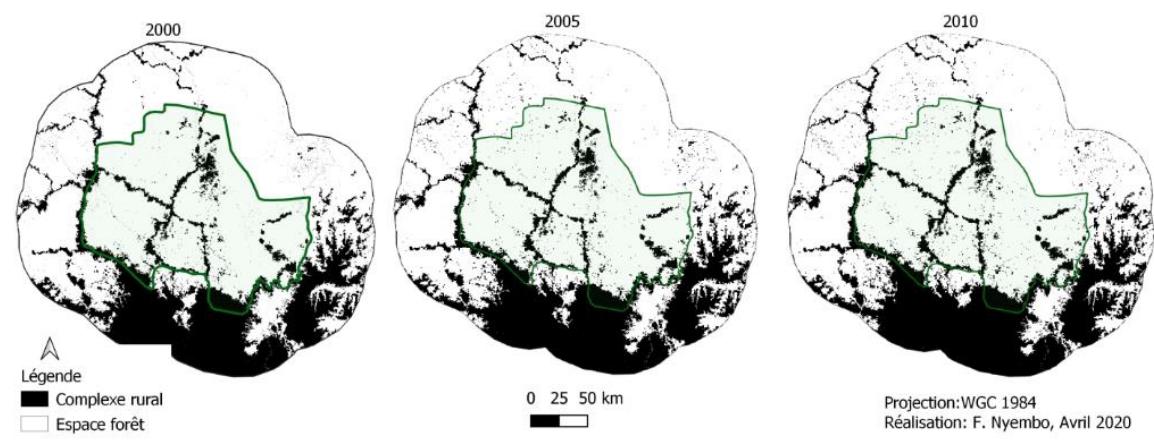

Figure 3 : Dynamique de la distribution du complexe rural dans une zone tampon estimée de $50 \mathrm{~km}$ Données (Forest Cover Dynamics of Shifting Cultivation in the DRC 2000-2010)

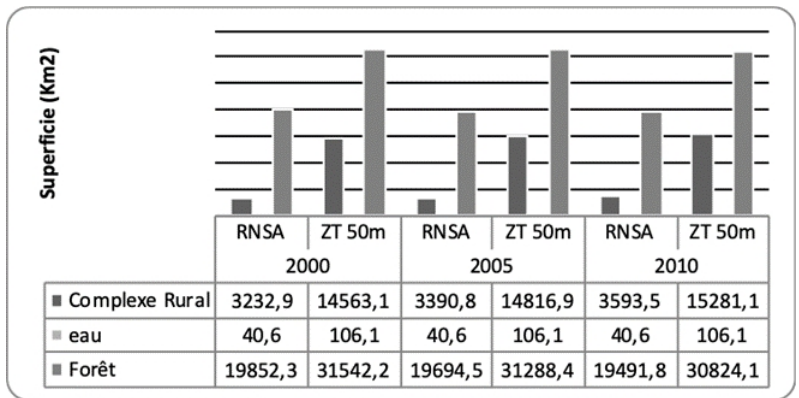

Figure 4 : Dynamique de la superficie du complexe rural dans la RNSA 2000-2010

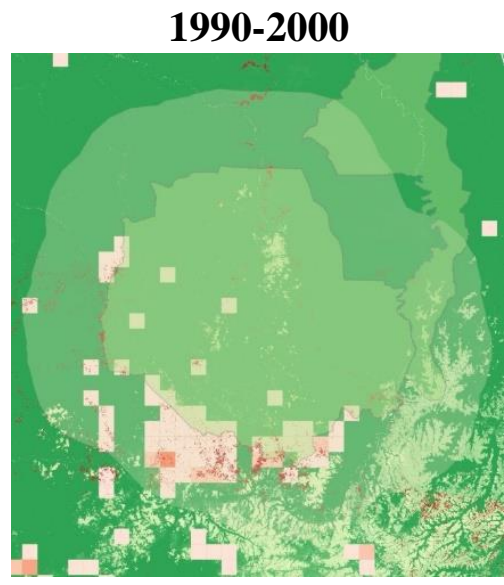

2010-2014

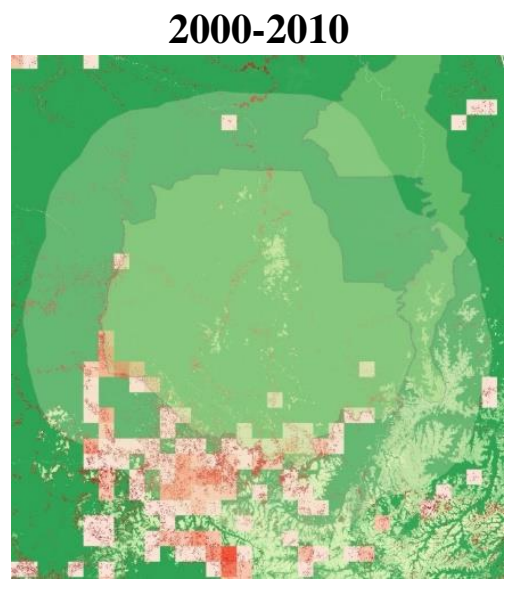

2014-2018 

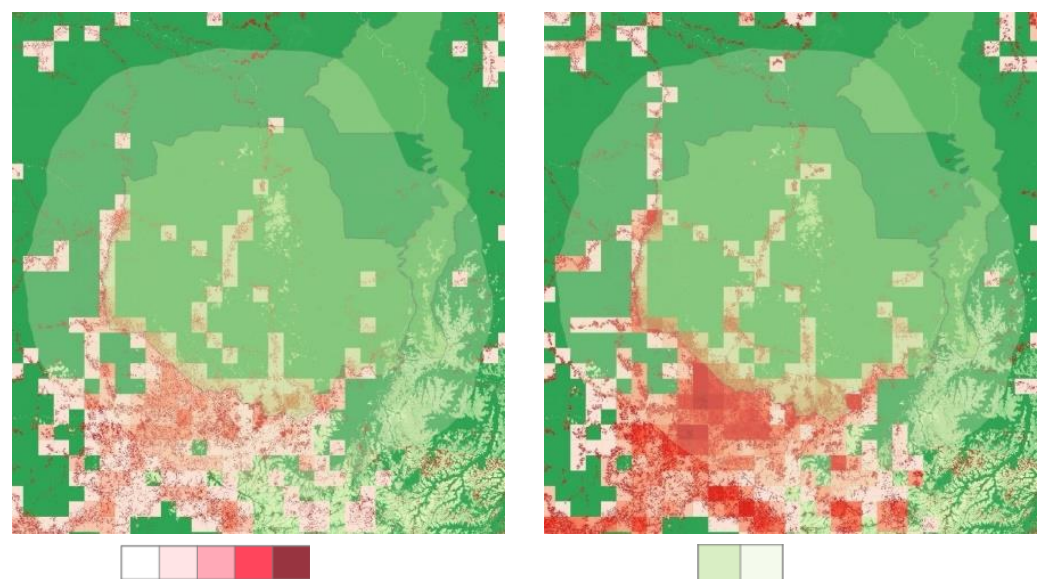

Basse

Elevée

Zone protégée Zone tampon

Figure 5 : Distribution spatiale de l'intensité de la pression sur le couvert forestier (Données : Forest cover change 1990 - 2010 - 2014-2018)

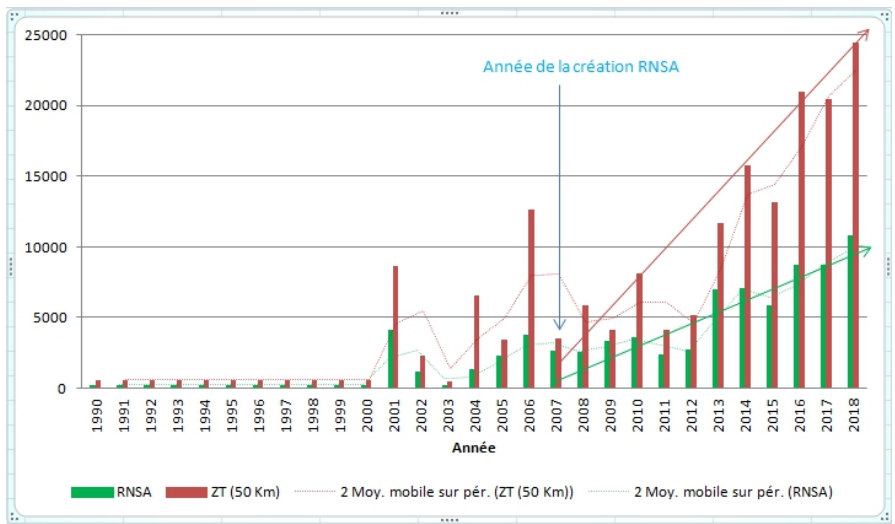

Figure 6: Perte annuelle du couvert forestier dans la RNSA et dans la Zone tampon de 1990 à 2018

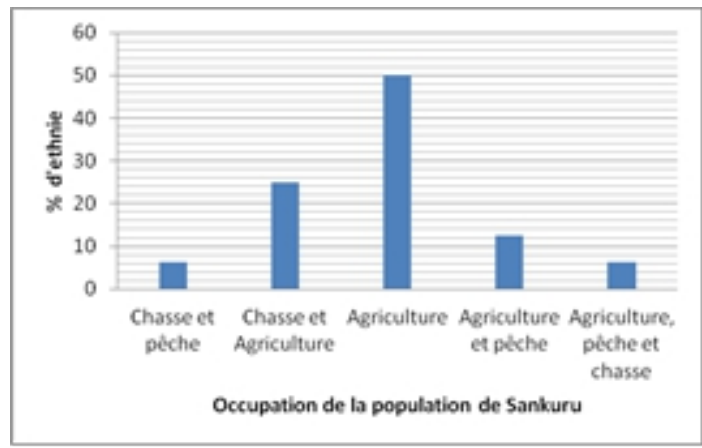

Figure 7 : Proportion d'ethnies par type d'occupation à Sankuru 


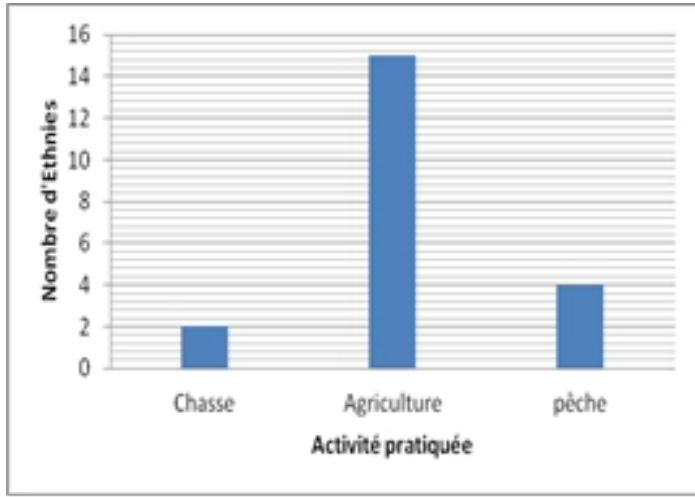

Figure 8 : Nombre d'ethnies par type d'activités à Sankuru

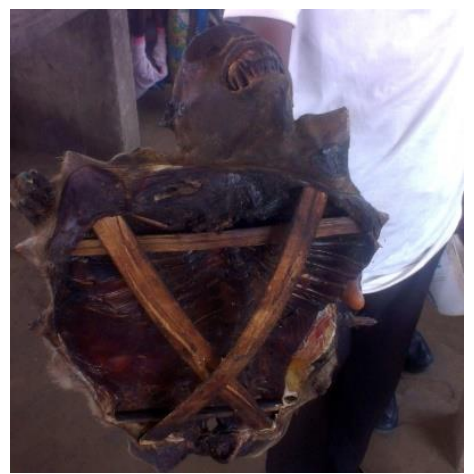

Photo 1 : Carcasse fumée du bonobo sur le marché de Lomela à Sankuru (Photo. F. Nyembo, 2018)

\section{Faible niveau d'application de la loi dans la RNSA}

L'arrêté ministériel portant sur la création de la RNSA, contient la déclaration selon laquelle « dans le cadre d'une conservation communautaire participative, la RNSA fait l'objet de zonage, après concertation entre l'ICCN et toutes les parties prenantes particulièrement les communautés locales et les peuples autochtones ». Malgré l'arrêté, la concertation semble n'avoir jamais abouti à un consensus sur les parties consacrées exclusivement à la conservation. Depuis l'entrée en vigueur de cet arrêté en date du 06 novembre 2007, son exécution reste en souffrance. En effet, les activités clairement interdites continuent sans que les auteurs soient inquiétés. Les enquêtes ont montré que $80 \%$ de la population vivant aux alentours de la RNSA trouvent leur bois de feu à l'intérieur de la forêt en général parmi laquelle $6,6 \%$ reconnaissent avoir souvent pénétré la réserve pour récolter le bois. $88 \%$ de la population dit ne pas être au courant de l'existence d'une quelconque mesure à respecter vis-à-vis de la forêt dans leur milieu. 73,33\% ont répondu par « non » et disent ne pas savoir non seulement là où la RNSA se localise mais ignore aussi son existence. 


\section{Faible niveau d'engagement des parties prenantes}

Au niveau de la chefferie de Djonga, les autorités coutumières sous pression des chasseurs du village refusent de collaborer ouvertement avec le Ministère de l'Environnement Conservation de la Nature et Développement Durable (MECNDD); la population locale et le peuple autochtone ne considèrent pas la RNSA comme étant une aire protégée. Leur engagement en matière de conservation dans cette localité profonde de la RDC est très faible. Aucun projet de conservation n'a réussi à démarrer au sein de la réserve. Une situation qui ne permet pas aux chercheurs et aux autorités politicoadministratives de donner une estimation réaliste de la population de bonobos encore présente au sein de la RNSA.

Par rapport à la situation générale du pays, la RNSA semble pour l'instant épargnée par des permis miniers au sein de son aire, contrairement à d'autres aires protégées du pays ; ce constat est le même pour les concessions forestières agréées (Figure 9).

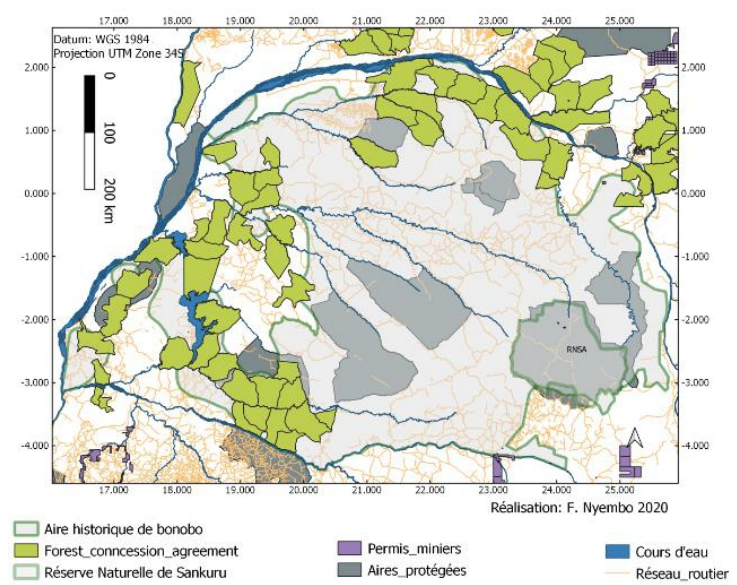

Figure 9 : Carte des aires protégées superposée au permis miniers et concessions forestières.

\section{Discussion}

La déforestation est la menace qui tire plus d'attention à Sankuru. L'agriculture sur brûlis, l'expansion de villages, le manque d'alternatives de substance et le faible niveau d'application de la loi constituent les plus principaux moteurs de la perte de l'habitat du bonobo. Les différentes données de changement du couvert forestier utilisées indiquent que la majeure partie de la déforestation de Sankuru des 20 dernières années s'est concentrée près des villes, ainsi que le long des routes et des voies qui traversent la réserve. Cela explique l'élan avec lequel le complexe rural s'est étendu vers le nord de Sankuru conduisant rapidement à la fragmentation de l'habitat ainsi que la réduction de la disponibilité des plantes utilisées comme ressource alimentaire par le singe en général. Plusieurs points de vue existent sur l'influence du couvert végétal sur la présence de grand singe. Narat et al. (2012) n’ont pas 
observé une corrélation positive entre le nombre d'arbres en fruits et les indices de leur présence pour les bonobos particulièrement d'Embinima, tandis que Maloueki et al. (2013) ont trouvé que le nombre d'individus de bonobos tend à augmenter à mesure que la végétation boisée croît. Dans tous les cas, la préservation de l'écosystème forestier joue un grand rôle dans le maintien de la vie et nécessite d'être constamment suivi. Kissinger et al. (2012) montrent que dans les zones tropicales, l'agriculture paysanne est la principale cause de déforestation. Elle contribue à $35 \%$ à la destruction de la forêt en Afrique.

L'Analyse spatiale du changement de couvert végétal montre que la forêt recule de $0,15 \%$ par an au sein de la RNSA, un taux inférieur à la moyenne nationale de déforestation dont le taux est estimé à 0,22\% par an (De Wasseige et al., 2012), mais proche de 0,18\% trouvé dans des conditions semblables à Yangambi autour de la même période (Kyale et al., 2019). Le résultat révèle que l'agriculture est pratiquée par $93 \%$ des ethnies retrouvés dans et autour de Sankuru. La moitié de la population pratique exclusivement l'agriculture et l'autre moitié la combine soit avec la pêche soit avec la chasse. Ainsi, l'agriculture itinérante sur brûlis et la production du bois-énergie sont les principales causes de la déforestation dans le Sankuru. Cette observation marche de paire avec le constat fait au nord du grand Kasaï (Kabata, 2018). Les données du Service National de Vulgarisation (SNV), publiées en 1994, montre clairement que l'agriculture est une habitude ancrée dans la population de Sankuru, depuis plusieurs décennies (MECNT, 1999; DSRP, 2005). Cela pourrait justifier la forte expansion du complexe rural à une vitesse d'environ 3605 ha/an.

Certains auteurs contestent l'idée selon laquelle l'agriculture itinérante sur brûlis est responsable d'une déforestation irréversible et d'une dégradation catastrophique des forêts et des sols des régions tropicales (Tchatchou et al., 2015), ce qui pourrait réduire l'attention de gestionnaire sur cette menace. Cependant, Molinario et al. (2015) soutenue par Ickowitz et al. (2015) ont démontré avec succès la complexité des relations qui existent entre la culture itinérante et les impacts sur la forêt. Molinario et ses collègues ont constaté qu'il y a une déconnexion dans la manière dont les gens abordent le problème de la déforestation dans le bassin du Congo et particulièrement en R.D.C. Ils ont suggéré la prise en compte de la notion du complexe rural pour mieux apprécier l'impact. L'intégration de ce contexte dans l'évaluation de la dégradation ôte à l'agriculture sur brûlis le caractère bénin et non perturbateur au sens écologique tel que prôné par certains ethnobotanistes et anthropologues (Dounias et al., 2000).

Etant donné que la recherche de la nourriture essentiellement constituée des produits des arbres et des végétaux herbacés terrestres dicte le déplacement des différents groupes de bonobos (Inogwabini et matungila, 
2009), il est à craindre que l'absence du contrôle sur l'agriculture amplifie les trouées qui existent déjà dans la couverture forestière au sein de Sankuru, car une situation pareille produit des impacts sur l'écosystème forestier et l'habitat de la biodiversité (Molinario et al., 2017; Molinario et al., 2020). Cela pourrait davantage pousser le bonobo à se déplacer vers le milieu où les conditions sont meilleures pour sa survie (Anderson et Ohmart 1986; IUCN et ICCN, 2012). A Sankuru, cette situation s'est déjà produite. Cependant, c'est la chasse qui a été d'une manière globale placée en première ligne de cause d'origine anthropique ayant conduit au déplacement et la diminution des effectifs des espèces emblématiques de Sankuru (Mulavwa et al., 2008).

Le braconnage est souvent cité comme la plus importante menace pour la survie du bonobo ; son impact sur les grands singes est plus direct et immédiat (Fruth et al, 2016). Cependant, certaines des causes sous jacentes au braconnage telle que l'instabilité politique (Gonedelé et al, 2006), la circulation des munitions et des armes à feu (Fruth et al., 2013) ont sensiblement diminué ces dernières années dans certaines parties de la RDC, particulièrement dans le centre du pays, notamment dans la province de Sankuru où les rebelles et les militaires régnaient en maîtres sur les forêts. Les différents rapports sur le braconnage (MECNT, 1999; Liengola et al., 2009; UICN et ICCN, 2012) montre que la position qu'occupe cette pratique dans la hiérarchie des menaces sur la faune sauvage est favorisée par des instabilités politiques. En 2016, le gouvernement avait reconnus ce problème en affirmant que les guerres (1996, 1998, 2003, 2012) en RDC ont été désastreuses pour la conservation de la biodiversité (MEDD, 2016). La force de l'impact de la chasse est due à sa capacité d'avoir un effet immédiat sur le groupe de Bonobo (Fruth et al., 2016). Cependant, les éléments de l'habitat tels que le couvert végétal, la composition des strates, la distribution et la densité des arbres et aussi la densité du feuillage jouent un rôle important dans la sélection de l'habitat par l'espèce (Anderson et Ohmart, 1986). Les bonobos nichent le plus souvent là où le couvert végétal en moyen est plus grand. Cela s'explique par le fait que les nids sont construits dans des arbres qui offrent un feuillage approprié pour construire un coussin confortable sur lequel dormir et également leur préférence des couches d'arbres qui fournissent une bonne couverture, pour éviter d'être détectés par des prédateurs potentiels durant la nuit (Inogwabini, 2015).

Le besoin urgent à Sankuru est la préservation de son intégrité. Dans la définition de l'aire protégée, Dudley (2008) précise que l'espace géographique doit être clairement défini, reconnu, consacré et géré, par tout moyen efficace, juridique ou autre (Mauvais et al., 2017). Ainsi, par rapport au résultat des analyses, il est approprié que les efforts de gestion dans l'aire protégée de Sankuru soient maximisés dans la lutte contre l'expansion du complexe rural au sein de la réserve. Sans un habitat favorable, il n y aura pas 
de bonobo. La vie de ce primate emblématique est liée au milieu forestier particulièrement les forêts denses humides, les forêts marécageuses, les forêts sèches, les forêts secondaires et les mosaïques forêt/savane (Reinartz et al., 2008). L'endémie de ce primate est liée à son aire de distribution historique où les conditions environnementales sont spécialement favorables à son tempérament. L'habitat du bonobo est, par conséquent, l'un des éléments de valeur que les gestionnaires de Sankuru sont invités à surveiller face aux différentes menaces d'origine anthropique ou climatique (Malhi et al., 2008).

\section{Conclusion et Recommandations}

Ce travail a permis de prioriser les menaces qui pèsent sur le bonobo au sein de la RNSA. Les résultats obtenus démontrent qu'à l'échelle de la réserve, la déforestation est une menace principale pour le bonobo. L'exploitation de plusieurs cartes de niveau national et mondial sur des périodes allant de 2000 à 2010 (autour de l'année de la création de la réserve) et de 1990 à 2018 (pour une vision plus large), ont permis de déterminer l'envergure spatiale de la déforestation à l'intérieur de la réserve ainsi qu'aux alentours de ses limites sur une largeur de $50 \mathrm{~km}$. Chaque année, la forêt régresse de $0,15 \%$ au profit de l'extension du complexe rural avec une vitesse de 3605 ha/an de 2000 à 2010. Le manque d'alternatives de subsistance maintien les autochtones et la population locale dans la pratique de l'agriculture sur brûlis. Cette dernière est largement pratiquée en tant qu'activité principale pour les populations de 15 ethnies sur 16 .

Plusieurs défis sont à relever pour sauvegarder l'habitat du bonobo au niveau de Sankuru. L'analyse spatiale a permis de voir que le complexe rural s'étend avec la même vitesse à l'intérieur et à l'extérieur de la limite de la RNSA, signe d'une indifférence et ignorance totale liée à la conservation de la nature. L'existence de la zone tampon ainsi que des détails sur sa dimension ne sont pas clairement mentionnés dans l'arrêté portant création de la RNSA.

La vente des carcasses de bonobo en provenance de Djonga continue de se faire en secret au marché de Lomela. C'est au marché que certains chasseurs apprennent qu'il existe des restrictions sur certaines espèces. Ces éléments démontrent que la gestion de la RNSA est encore très limitée. Cette situation laisse la porte grandement ouverte à toutes les menaces soulevées dans ce travail et qui sont toutes susceptibles de faire disparaitre le bonobo dans son habitat de Sankuru.

Afin d'assurer à long terme la conservation de la nature et des services éco-systémiques et des valeurs culturelles qui lui sont associés (Mauvais et al., 2017), nous recommandons qu'une étude approfondie soit menée pour identifier les différentes zones refuges de bonobos au sein de la RNSA et y déterminer la taille de la population de ce grand singe. L'inventaire 
effectué en 2009 était très partiel (Liengola et al., 2009) et orienté uniquement dans des zones supposées contenir le Bonobo (UICN et ICCN, 2012). Nous encourageons l'Institut Congolais pour la Conservation de la Nature à préserver l'existence de cette aire protégée en formant particulièrement les communautés locales et les peuples autochtones sur la conservation communautaire participative. Le Ministère de l'Environnement et Développement Rural devrait mettre à la disposition de toutes les parties prenantes à la conservation, un statut juridique définissant clairement la délimitation et le mode de gestion de la zone tampon dans la RNSA.

\section{Remerciements}

Nous exprimons notre gratitude à l'Institut de Recherche pour le Développement (IRD France) pour l'Allocation de Recherche de Thèse au Sud (ARTS) accordée par le truchement de l'Unité Mixte de Recherche Espace DEV, maison de la télédétection de Montpellier. Nous remercierons également l'Université Félix Houphouët Boigny (UFHB) pour l'encadrement scientifique à travers le programme doctoral Changement Climatique, Biodiversité et Agriculture Durable (CCBAD) via le Centre d'Excellence Africain (CEA). Nous remercions l'Université Officielle de Mbujimayi pour toutes les autorisations de sortir accordées.

\section{References :}

1. Akaibe D. (2014). Etat Des lieux de la biodiversité dans la R.D. Congo, 1st International Conference on Biodiversity in the Congo Basin, 6-10 juin 2014, Kisangani, République Démocratique du Congo; Consortium Congo 2010 (l'Université de Kisangani, le Musée royal de l'Afrique centrale, l'Institut royal des Sciences naturelles de Belgique, le Jardin botanique Meise) et le Centre de Surveillance de la Biodiversité à Kisangani. 381p. http://congobiodiversityconference2014.africamuseum.be/fr/node/76

2. Anderson BW. et Ohmart RD. (1986). Inventaire et surveillance de l'habitat faunique. In: Copperider, AY, RJ Boyd \& HR Stuart (éd.). Inventory and Monitoring of Wildlife Habitat. U.S. Département américain de l'intérieur, Bureau of Land Management, Washington DC. pp. 639-660.

3. Chapman A.D. (2005). Les usages des données primaires d'occurrence d'espèces, version 1.0. Trad. Chenin, N. Copenhague : Global Biodiversity Information Facility, 103 p. Disponible en ligne sur http://links.gbif.org/gbif_usages_donnees_manual_fr_v1.1.pdf

4. Coxe S., Rosen N., Miller P., Seal U. (2000). Bonobo Conservation Assessment, Workshop Report. IUCN/SSC Conservation Breeding 
Specialist Group, Apple Valley, MN. Kyoto University Primate Research Institute, Inuyama, Japan. pp 21-22.

5. De Wasseige C., de Marcken P., Bayol N., Hiol Hiol F., Mayaux P., Desclée B., Billand A., Nasi R. (2012). Les forêts du bassin du Congo - État des forêts 2010. Luxembourg, Office des publications de l'Union européenne, 276p.

6. Doumenge C., Palla F., Scholte P., Hiol Hiol F., Larzillière A. (2015). Aires protégées d'Afrique centrale - État, 2015. OFAC, Kinshasa, République Démocratique du Congo et Yaoundé, Cameroun : 256p.

7. Dounias E. Tzerikiantz F., Carrière S., McKey D., Grenand F., Kocher-Schmid C., Bahuchet S. (2000). La diversité des agricultures itinérantes sur brûlis. APFT-ULB Bruxelles ; pp. 65-106

8. DSRP. (2005). Monographie de la province du Kasaï Oriental, Ministère du plan Unité de pilotage du processus DSRP Kinshasa / Gombe, République Démocratique du Congo, 145 p. https://ipamec.be/Documents/KASAI-ORI-DSRP.PDF

9. DUDLEY N. (2008). Lignes directrices pour l'application des catégories de gestion aux aires protégées. Gland, Union Internationale pour la Conservation de la Nature.

https://portals.iucn.org/library/efiles/documents/paps-016-fr.pdf

10. Eriksson J, Hohmann G., Boesch C., Vigilant L. (2004). Rivers influence the population genetic structure of bonobos population (Pan paniscus). Mol Ecol 13: 3425-3435.

11. Excel (2016). Microsoft Official Academic Course MICROSOFT EXCEL, Microsoft Office Specialist MOS 77-727. 282p; https://campus.uvci.edu.ci/certification/Excel_2016.pdf

12. FAO. (2020). FAOLEX Database: Arrêté $n^{\circ}$ 045/CAB/MIN/ECN$\mathrm{EF} / 03 / \mathrm{PDB} / 07$ du 06 novembre 2007 portant création d'une Réserve Naturelle dénommée "Réserve Naturelle du Sankuru" "RNSA "., consulté le 21 Mai 2020 ;

http://www.fao.org/faolex/results/details/en/c/LEX-FAOC085726

13. Fruth B., Hickey JR, André C., Furuichi T., Hart J., Hart T., Kuehl H., Maisels F., Nackoney J., Reinartz G., Sop T., Thompson J., Williamson EA. (2016). Pan paniscus (version publiée en 2016 errata). La Liste rouge UICN des espèces menacées 2016. 18p. Téléchargé le 13 Janvier 2020.

e.T15932A102331567. https://dx.doi.org/10.2305/IUCN.UK.20162.RLTS.T15932A17964305.en.

14. Fruth B., Williamson,E.A., Richardson M.C. (2013). Bonobo Pan paniscus. In: R.A. Mittermeier, A.B. Rylands and D.E. Wilson (eds), Handbook of the Mammals of the World Volume 3: Primates, pp. 853854. Lynx Edicions, Barcelona, Spain. 
15. Mauvais G., Chataigner B., Panaretos V.P., Hema E.M. et Koné I. (2017). Gestion des aires protégées en Afrique: suivi écologique, (C) EPFL Press, 2017; ISBN 978-2-88914-421-1, 47 p.

16. Gonedelé Bi.S., Dietmar Z., Koné I., Goné bi Z.B., Akpatou K.B., Koffi B. J-C. , Sangaré A. and Boesch C. (2006). A West African Black-and-White Colobus Monkey, Colobus polykomos dollmani, Facing Extinction; Primate Conservation (21): 55-61; http://www.bioone.org/doi/full/10.1896/0898-6207.21.1.55

17. GRASP. (2005). A summary of the intergovernmental meeting on great apes and the fist meeting of the council of the great apes survival project in Kinshasa, Democratic Republic of the Congo (DRC). Published by the International Institute for Sustainable Development (IISD) Volume 113, No.1; 9 p. https://enb.iisd.org/download/pdf/sd/ymbvol113numle.pdf

18. Hansen M., Roy D., Lindquist E., Justice C., Altstatt A. (2008). Une méthode d'intégration des données MODIS et Landsat pour la surveillance systématique du couvert forestier et des changements en Afrique centrale Télédétection de l'environnement, v112 : 2495-2513.

19. Hansen MC., Potapov PV., Moore R., Hancher M., Turubanova SA., Tyukavina A., Thau D., Stehman SV., Goetz SJ., Loveland TR., Kommareddy A., Egorov A., Chini L., Justice CO., Townshend JRG. (2013). «Cartes mondiales à haute résolution du changement du couvert forestier du 21e siècle». Science 342 (15 novembre): 85053. Données disponibles en

ligne. http://earthenginepartners.appspot.com/science-2013-globalforest .

20. Hansen MC., Krylov A., Tyukavina A., Potapov PV., Turubanova S., Zutta B., Ifo S., Margono B., Stolle F., Moore R. (2016). Humid tropical forest disturbance alerts using Landsat data. Environmental Research Letters, 11 (3). Accessed through Global Forest Watch on October 23, 2019. www.globalforestwatch.org.

21. Ickowitz A., Slayback D., Asanzi P., Nasi R. 2015. Agriculture and deforestation in the Democratic Republic of the Congo: A synthesis of the current state of knowledge. Occasional Paper 119. Bogor, Indonesia: CIFOR. 28 p. https://doi.org/10.17528/cifor/005458

22. Inogwabini B.-I. (2015). The Bonobo Pan paniscus (Mammalia: Primates: Hominidae) nesting patterns and forest canopy layers in the Lake Tumba forests and Salonga National Park, Democratic Republic of Congo. Journal of Threatened Taxa 7(12): 78537861; https://doi.org/10.11609/JoTT.o4217.7853-61

23. Inogwabini B-I et Matungila B. (2009). Bonobo Food Items, Food Availability and Bonobo Distribution in the Lake Tumba Swampy 
Forests, Democratic Republic of Congo. The Open Conservation Biology Journal. https://doi.org/10.2174/1874839200903010014

24. Kabata K. (2018). Pouvoir, territorialité et conflictualité au Grand Kasaï (République démocratique du Congo). Belgeo [En ligne], 2 | 2018, mis en ligne le 17 septembre 2018, consulté le 30 avril 2019. URL: http://journals.openedition.org/belgeo/26916

25. Kissinger G., Herold M., De Sy V. (2012). Drivers of Deforestation and Forest Degradation: A Synthesis Report for REDD+ Policymakers. Lexeme Consulting, Vancouver Canada, August 2012.

26. Kyale J., Wardell A., Mikwa J-F., Kabuanga J-M., Monga M., Oszwald J., Doumenge C. (2019). Dynamique de la déforestation dans la Réserve de biosphère de Yangambi (République démocratique du Congo) : variabilité spatiale et temporelle au cours des 30 dernières années. Bois et Forêts des Tropiques, 341 : 15-28. Doi : https://doi.org/10.19182/bft2019.341.a31752

27. Liengola, I., Vosper, A., Maisels, F., Bonyenge, A., Nkumu, P. (2009). Conserving Bonobos in the Last Unexplored Forest of the Democratic Republic of Congo-the Tshuapa-Lomami-Lualaba Landscape. Rapport non publié, Wildlife Conservation Society, New York

28. Malhi Y., Roberts J.T., Betts R.A., Killeen T.J., Li W.H., Nobre C.A. (2008). Climate change, deforestation, and the fate of the Amazon. Science 319: 169-172.

29. Maloueki U., Kumugo N., Mukulire M., Bekeli N. (2013) Estimation de la densité par comptage des nids des Bonobos (Pan paniscus) dans la région de Bolobo des localités de Nkala et Embirima, République Démocratique du Congo: résultats préliminaires1. Revue de primatologie. DOI: $10.4000 /$ primatologie. 1660

30. MECNT. (1999). Plan d'action provinciaux de la biodiversité ; appendice du plan d'action national 1986 : Etude régionale pour la planification Agricole de 1986 Etude régionale pour la planification Agricole.

31. MEDD. (2016). Stratégie et plan d'action nationaux de la biodiversité (2016-2020), Ministère de l'Environnement, Conservation de la Nature et Développement Durable de la République Démocratique du Congo; 91p. http://extwprlegs1.fao.org/docs/pdf/Cng169379.pdf

32. Mertens, B. Orekan, V. (2019). Actes de la Conférence «Des images satellites pour la gestion durable des territoires en Afrique », 13-15 mars 2019, Cotonou, Bénin.

33. Mittermeier R.A., Brooks T., Fonseca G., Brito D. (2008). « Hotspots et régions sauvages. Les pays de mégadiversité. »In Jacquet P., Tubiana L. : L'annuel du développement durable - Regards sur la 
Terre 2008, Paris, Les Presses de Sciences Po, Annuels : 131-133; 153-154.

34. Molinario G., Hansen M C., Potapov P V. (2015): Forest cover dynamics of shifting cultivation in the Democratic Republic of the Congo 2000-2010. Environmental Research Letters, Volume 10, Number 9; (Environ. Res. Lett. 10 094009)

35. Molinario G., Hansen M. C., Potapov P. V., Tyukavina A., Stehman S., Barker, B., Humber, M. (2017). Quantification of land cover and land use within the rural complex of the Democratic Republic of Congo. Environmental Research Letters, 12(10), 104001.

36. Molinario G., Hansen M. C., Potapov P. V., Tyukavina A., Stehman S. (2020). Contextualizing Landscape-Scale Forest Cover Loss in the Democratic Republic of Congo (DRC) between 2000 and 2015. Land 2020, 9, 23; doi:10.3390/land9010023.

37. Mulavwa M., Furuichi T., Yangozene K., Yamba-Yamba M., Motema-Salo B., Idani G., Ihobe H., Hashimoto C., Tashiro Y., Mwanza N. (2008). Seasonal Changes in Fruit Production and Party Size of Bonobos at Wamba. pp. 121-134. doi:10.1007/978-0-38774787-3_7

38. Muyaya B.K., Rudant J.P., Lumbuenamo R., Beland M., and Riera B. (2016). "Spatial dynamic of the hunting area and Bombo Lumene reserve between 2000 and 2015 by optical satellite imagery". International Journal of Innovation and Applied Studies 18( 2): 559568.

39. Narat V., Marie C., Bokika N.JC., Dumez R., Krief S. (2012). «Etude préliminaire de l'influence des disponibilités alimentaires et des activités humaines sur l'utilisation spatiale de l'habitat par les chimpanzés et les bonobos », Revue de primatologie 25p. http://journals.openedition.org/primatologie/1121

40. Nyembo F.K., Lusa R.M, Muanza A.K, Mukadi A.N., Mamadou C., Kamutanda K., Koné I. (2019). Couvert forestier et distribution de Pan paniscus dans la Réserve Naturelle de Sankuru, RD Congo. Conférence OSFACO : Des images satellites pour la gestion durable des territoires en Afrique, Mar 2019, Cotonou, Bénin. 18p; https://hal.archives-ouvertes.fr/hal-02189507/document

41. Oliveau S. (2011). "L'espace compte! Mesurer les structures spatiales du changement social », Université d'Aix-Marseille 1, 86p .

42. QGIS. (2020). User Guide Version 3.4 ; GGIS Project, Licence GNU de documentation libre, 641p. Copyright (c) 2004 - 2017 QGIS Development Team; https://www.qgis.org

43. Tchatchou B, Sonwa DJ, Ifo S., Tiani AM. (2015). Déforestation et dégradation des forêts dans le Bassin du Congo : État des lieux, causes 
actuelles et perspectives. Papier occasionnel 120. Bogor, Indonesie: CIFOR. 60p.

44. Thompson J., Hohmann G., Furuichi T. Bonobo Workshop: Behaviour, Ecology and Conservation of Wild Bonobos. Workshop Report, Primate Research Institute, Kyoto University, Inuyama, Japan.

45. Thompson-Handler, N., Malenky, R.K., Reinartz, G.E. (1995). Action Plan for Pan paniscus: Report on Free Ranging Populations and Proposals for their Preservation. Zoological Society of Milwaukee, Milwaukee, WI.

46. UICN \& ICCN. (2012). Bonobo (Pan paniscus): Stratégie de Conservation 2012-2022. Gland, Suisse: Groupe de spécialistes des primates de la CSE/UICN \& Institut Congolais pour la Conservation de la Nature $68 \mathrm{p}$.

47. Vos J.M., Joppa L.N., Gittleman J.L., Stephens P.R. et Pimm S.L. (2014). Estimating the normal background rate of species extinction. Conservation Biology, 29(2), pp. 452-462. Repéré à : http://onlinelibrary.wiley.com/doi/10.1111/cobi.12380/abstract;jsessi onid=754B5F9D97D4C56F83802ECC580187A3.f04t02 